Subtyping of $T$ pallidum, using a previously-published technique, was based on the molecular characterisation of two variable treponemal repeat genes, arp and tpr.

Results Macrolide resistance profiles and the subtype distribution for all 60 DNA samples were obtained. The mean age of all participants was 27.4 years (range 18-43; SD 5.24) of which 55 (92\%) were male and $5(8 \%)$ were female. None of the samples analysed contained the $23 \mathrm{~S}$ rRNA gene point mutation that confers macrolide resistance. A total of eight arp repeat sizes, 8 RFLP patterns and a combined total of 17 subtypes were identified in this study population. The most common subtypes were $14 \mathrm{~d}(43 \%)$, followed by $17 \mathrm{~d}(13 \%), 14 \mathrm{~b}(7 \%), 22 \mathrm{~b}(5 \%)$ and $23 \mathrm{~b}(5 \%)$.

Conclusions This is the first South African study to examine both macrolide-resistance profiles and the subtype distribution of syphilis strains using molecular techniques. Macrolide-resistant $T$ pallidum strains appear to be uncommon in South Africa compared to more developed countries. Our results indicated subtype $14 \mathrm{~d}$ to be the predominant circulating $T$ pallidum strain in South Africa and there seem to be a high degree of genetic heterogeneity within this population.

\section{P4-S3.02 SUBTYPING OF TREPONEMA PALLIDUM STRAINS BY SEQUENCE ANALYSIS OF TP0279 AND TP0548}

doi:10.1136/sextrans-2011-050108.517

${ }^{1} \mathrm{~A}$ Pillay, ${ }^{1} \mathrm{C}$ Y Chen, ${ }^{2} \mathrm{M}$ G Morshed, ${ }^{3} \mathrm{~S}$ Philip, ${ }^{1} \mathrm{R} \mathrm{C}$ Ballard. ${ }^{1} \mathrm{CDC}$, Atlanta, USA; ${ }^{2}$ British Columbia Centre for Disease Control Public Health Microbiology and Reference Laboratories, Atlanta, USA; ${ }^{3}$ STD Prevention and Control Services, San Francisco Department of Public Health, San Francisco, USA

Background The goal of the study was to evaluate two sequencebased subtyping methods for their ability to further differentiate Treponema pallidum strains characterised by the CDC typing system.

Methods 12 clinical specimens with the $14 \mathrm{~d}$ strain type obtained from patients with GUD in Cape Town in 2000; 14 specimens with the $14 \mathrm{~d}$ strain type from patients with primary or secondary syphilis in San Francisco collected between 2004 and 2007; and 12 clinical specimens with the $14 \mathrm{~d}$ strain type collected between 2000 and 2002 from a syphilis outbreak in Vancouver were included in the study. Specimens were previously characterised using the CDC typing method which involves analysis of the sequence variability with tprE, G, and $J$ and, the variable number of 60-bp tandem repeats within the arp gene. Subtyping was performed by PCR amplification of a homonucleotide G" tandem repeat within tp0279 and a variable region within tp0548. PCR amplicons were purified and directly sequenced using the BigDye ${ }^{\circledR}$ Terminator v3.1 cycle sequencing kit and an $\mathrm{ABI} 3130$ sequencer.

Results Of the 12 samples from South Africa, sequence analysis of tp0279 revealed three subtypes (9G, 10G, 11G) and combining this data with the CDC typing method produced strain subtypes $14 \mathrm{~d} 9$, $14 \mathrm{~d} 10$, and $14 \mathrm{~d} 11$. Sequence analysis of the variable region within tp0548 resulted in five subtypes designated a, c, f, k, and 1 among these samples and, combination with strain type using the CDC method produced subtypes 14d/a, 14d/c, 14d/f, 14d/k, 14d/1. All 14 samples from San Francisco had 9G tandem repeats within tp0279 resulting in subtype $14 \mathrm{~d} 9$. Sequence analysis of the variable region within tp0548 resulted in 3 subtypes designated $f, g$ and $j$ among these 14 samples and incorporation of the CDC typing method produced subtypes $14 \mathrm{~d} / \mathrm{f}, 14 \mathrm{~d} / \mathrm{g}$, and $14 \mathrm{~d} / \mathrm{j}$. Of the 12 samples from Vancouver, sequence analysis of tp0279 produced two subtypes (8G,
9G) resulting in strain subtypes $14 d 8(1 / 12)$ and14d9 (11/12). Sequencing of tp0548 also produced two subtypes $(e, f)$ resulting in strain subtypes $14 \mathrm{~d} / \mathrm{e}(1 / 12)$ and14d/f (11/12).

Conclusions The tp0279 subtyping method further differentiated $14 \mathrm{~d}$ strains into four subtypes while the tp0548 method differentiated $14 \mathrm{~d}$ strains into eight subtypes. Subtyping of strains from the initial syphilis outbreak in Vancouver suggests clonal spread of $T$ pallidum. Both subtyping methods enhanced the original CDC typing method and appear to be promising tools for molecular epidemiological studies on syphilis.

\section{P4-S3.03 CHARACTERISATION OF UREAPLASMA PARVUM FROM SYMPTOMATIC AND ASYMPTOMATIC MEN ATTENDING A FAMILY PRACTICE IN PRETORIA, SOUTH AFRICA}

doi:10.1136/sextrans-2011-050108.518

${ }^{1} \mathrm{M}$ Kock, ${ }^{2} \mathrm{~N}$ Maningi, ${ }^{3} \mathrm{~A}$ Adam, ${ }^{1} \mathrm{~A}$ Hoosen. ${ }^{1}$ University of Pretoria, National Health Laboratory Service, Pretoria, South Africa; ${ }^{2}$ University of Pretoria, South Africa; ${ }^{3}$ Private practitioner, Pretoria, South Africa

Background The genus Ureaplasma colonises human mucosal surfaces such as urogenital tract of men and women. In men it has been implicated in the aetiology of non-gonococcal urethritis and infertility. Although its pathogenesis is not yet fully understood, it has been suggested that certain serotypes are associated with disease. This study undertook to detect genital Ureaplasma spp. and to characterise Ureaplasma parvum in men with and without urogenital symptoms.

Methods Two hundred first void urine specimens were collected from symptomatic (100) and asymptomatic (100) men attending a private clinic. All specimens were cultured in U9 broth and subcultured on A2 agar medium for confirmation. All isolates were tested for susceptibility using the Mycofast Evolution 3 kit. DNA was extracted from all specimens and amplified using a multiplex TaqMan polymerase chain reaction assay targeting the multiple-banded antigen gene for the detection and serotyping of $U$ parvum. Ureaplasma urealyticum was detected by a commercial real-time PCR kit.

Results Cultures were positive in 16/100 symptomatic and 12/100 asymptomatic men $(p=N S)$. All isolates were susceptible to doxycycline, pristimycin, roxycycline and azithromycin. One Ureaplasma spp. from an asymptomatic male was resistant to ciprofloxacin and josamycin and intermediately resistant to ofloxacin and another was resistant to ofloxacin. An isolate from a symptomatic man was resistant to ciprofloxacin. There was no significant difference $(p=0.16)$ between the $U$ parvum isolated from symptomatic (11) $100)$ and asymptomatic (18/100) men as well as for $U$ urealyticum from symptomatic $16 / 100$ and asymptomatic $15 / 100$ men ( $p=0.86$ ). Four men (two from each group) were colonised by both Ureaplasma spp.. The predominant serotype was six, followed by types 1,14 and 3 with no significant difference between symptomatic and asymptomatic men $(p=0.309)$.

Conclusions There is no data of circulating U parvum serotypes from South Africa. The prevalence rate was low and no significant differences were found between symptomatic and asymptomatic men for both Ureaplasma spp.. Serotype 6 was the most common type compared to reports from developed countries which suggests type 3 as being the most common. Macrolides and tetracyclines remain effective drugs for treatment of the Ureaplasma infections. Molecular techniques are valuable identification and characterisation of this fastidious group of bacteria. 